\title{
Feasibility of postpartum placement of the levonorgestrel intrauterine system more than six hours after vaginal birth
}

\author{
Gretchen S. Stuarta ${ }^{a},{ }^{,}$, Amy G. Bryant ${ }^{a}$, Erica O'Neilla, and Irene A. Doherty ${ }^{b}$ \\ aDepartment of Obstetrics and Gynecology, University of North Carolina at Chapel Hill, Chapel \\ Hill, NC, 27599 \\ bDivision of Infectious Diseases, Department of Medicine, University of North Carolina at Chapel \\ Hill, Chapel Hill, NC, 27599
}

\begin{abstract}
Background-The objective of this study was to determine the feasibility of postpartum LNGIUS placement on the postpartum ward.

Study Design-This case-series study took place in a teaching hospital in North Carolina. Women were followed for six months and data on method satisfaction, study design satisfaction and expulsion were collected. Descriptive statistics were used.
\end{abstract}

Results-Forty women enrolled. Twenty-nine women (73\%) received the LNG-IUS at a median of $20 \mathrm{~h}$ (range 7-48h) after delivery, and all reported they would recommend this method of contraception to a friend. Eleven women had a spontaneous expulsion [38\% (95\% confidence interval 21,58)].

Conclusion-Placement of LNG-IUS more than $6 \mathrm{~h}$ postpartum was acceptable to women in this study. The expulsion rate of 38\% had statistical instability and should be interpreted with caution. However, our report may assist with individual counseling of women interested in postpartum LNG-IUS placement, or in future study designs.

\section{Keywords}

Contraception; postpartum; intrauterine device

\section{Introduction}

\begin{abstract}
Unintended pregnancy occurs in up to 50\% of all pregnancies in the United States [1]. The immediate postpartum period, after a birth but prior to discharge from the hospital, is an important, but underutilized time frame to initiate contraceptives, especially long acting contraceptives such as the subdermal implant or the intrauterine device (IUD) [2,3]. Women are often motivated to prevent or delay another pregnancy, they have immediate access to health care providers, they are known not be pregnant, and they may have uterine anesthesia in place. Postpartum intrauterine device (IUD) placement in particular may increase
\end{abstract}

\footnotetext{
(C) 2011 Elsevier Inc. All rights reserved

*Corresponding author: Gretchen S. Stuart, MD, MPHTM Department of Obstetrics and Gynecology, UNC School of Medicine, CB \#7570 Chapel Hill, NC 27599-7570 Telephone: (919) 843-9546, Gstuart@med.unc.edu.

Publisher's Disclaimer: This is a PDF file of an unedited manuscript that has been accepted for publication. As a service to our customers we are providing this early version of the manuscript. The manuscript will undergo copyediting, typesetting, and review of the resulting proof before it is published in its final citable form. Please note that during the production process errors may be discovered which could affect the content, and all legal disclaimers that apply to the journal pertain.
} 
utilization of postpartum contraception because up to $45 \%$ of women who state a desire to use the IUD postpartum may never receive one due to failure to return for the postpartum visit, early repeat pregnancy, or financial barriers $[3,4]$.

Although postpartum placement of the Copper T380A IUD (CuT380A) within $48 \mathrm{~h}$ of birth is condoned in national and international family planning practice guidelines, there are limited data on placement of the levonorgestrel intrauterine system (LNG-IUS) more than a few hours after birth [5,6]. Recent data from the United States indicate that postplacental (within 10 min of placental delivery) LNG-IUS placement is acceptable to women, but is also associated with spontaneous expulsions occurring in 10-28\% of women at 6 months [7,8]. Dahlke et al. [8] reported their experience of placing the LNG-IUS 30 min postplacentally in the delivery room, and they reported an expulsion rate of $24 \%$. An important limiting factor to increased uptake of immediate postpartum IUD insertion are older, but highly variable, data reporting expulsion rates of the CuT380A from as low as 5\% to as high as $70 \%[9,10,11]$. Limited data exist on LNG-IUS insertion more than a few hours of birth, and in a location other than the delivery room.

The advantages to immediate postpartum IUD insertion justify further investigation to accurately define the associated rates of IUD expulsion, and IUD use, at six months postpartum. For instance, this timing may be well suited for women who did not receive complete family planning counseling prior to delivery. In busy clinical settings, the clinician who is most skilled at postpartum insertion may not be available on the labor ward. Additionally, the infant status is known and the risk of postpartum hemorrhage has decreased. The primary objective of this study was to determine the feasibility of placing the LNG-IUS in women after a vaginal birth, and after transfer to the postpartum ward, but before discharge from the hospital. The secondary objectives were to assess the feasibility of our study design to inform future studies of immediate postpartum LNG-IUS insertion. We focused on the LNG-IUS in this feasibility study for two reasons: in our hospital the LNGIUS is more popular, and when we embarked upon this study there were no reported data on postpartum placement of the LNG-IUS beyond the postplacental timeframe.

\section{Methods}

This was a prospective case-series that took place at the University of North Carolina at Chapel Hill from July 2009 through October 2010. The Institutional Review Board of the University of North Carolina at Chapel Hill approved the study. We obtained an FDA IND (No.104, 220) and registered the study at Clinical Trials.gov (NCT00997932).

All women were recruited from the UNC Women's Hospital Obstetrics prenatal clinics and were patients of residents and faculty. Women who attended prenatal care at University of North Carolina's Women Hospital and who intended to use the LNG-IUS after the birth of their infant were invited to enroll. Eligibility criteria included age 18-40 years and currently pregnant with a singleton gestation of at least 27 completed weeks estimated gestational age, and with no known complications of pregnancy including, but not limited to, preeclampsia, non-gestational diabetes, and anemia. English- and Spanish-speaking women were enrolled. Additional criteria included anticipated vaginal delivery, no intention to leave the area for 7 months after enrollment, no known uterine anomalies, no known allergies to any components of the LNG-IUS, no history of cesarean delivery, no treatment of pelvic inflammatory disease within three months prior to the start of the pregnancy, no medical or personal conditions that in the judgment of study staff precluded participation in the study, and no contraindications to use of the LNG-IUS. 
After delivery, participants were interviewed on the postpartum floor and their medical records were reviewed to confirm none of the following secondary exclusions had occurred during labor or delivery: endometritis or chorioamnionitis during the intrapartum period; membranes ruptured for greater than $24 \mathrm{~h}$ prior to delivery; fever greater than or equal to $38^{\circ} \mathrm{C}$; the need to use additional medications other than oxytocin and/or misoprostol to control postpartum bleeding. Women who had any of the secondary exclusion criteria described above, or who declined further participation, left the study at this point. We based our selection of post-delivery exclusion criteria on the report by Hayes et al. [11] because that was the first report of LNG-IUS insertion after vaginal delivery based in the U.S.

Women who met the post-delivery criteria were brought to the treatment room on the postpartum ward for LNG-IUS placement by one of the authors (GSS, AGB or EO) using the technique described by O'Hanley at al. as a guide [12]. The clinician performed a bimanual exam and uterine sound to determine uterine size and cervical dilation, and the angle of the uterus to assess the angle of uterus and the uterine cavity. This information was used to identify whether placement by hand, inserter or ring forceps would be the best approach, and for assessment of uterine characteristics associated with expulsion.. We used either a speculum or we manually parted the vagina to visualize the cervix. The anterior lip of the cervix was grasped with a ring forcep to provide traction and stability for LNG-IUS placement. The LNG-IUS was grasped carefully and lightly to avoid crushing the device, along the stem of the device, at an angle, as described by O'Hanley et al. [12]. The LNG-IUS was then placed by one of the three investigators. All insertions were done under ultrasound guidance to confirm fundal placement. The LNG-IUS string was cut to the level of the vaginal introitus, or just proximal to the introitus.. Discomfort was assessed by asking women immediately after the LNG-IUS insertion to rate their pain on a scale of 1-10. All women who received the LNG-IUS received counseling regarding the possible increased risk of expulsion, and given detailed instructions regarding whom to call in the event of expulsion. An investigator was available $24 \mathrm{~h}$ a day to respond to phone calls.

Follow-up visits were planned at the 4- to 6-week postpartum visit, 3 months and 6 months after LNG-IUS placement. Data collected at each visit included satisfaction with the LNGIUS, whether or not the participants would recommend this method of contraception to a friend, and a pelvic exam to confirm presence or absence of the LNG-IUS string and an ultrasound examination to locate the LNG-IUS in the uterus. We also asked questions regarding participant willingness to be randomized to either timing or IUD type (LNG-IUS vs. CuT380A) in a future study.

Women who reported expulsion of the LNG-IUS were seen by an investigator and a report of expulsion was completed. Women received counseling regarding their need for alternative contraception.

We estimated proportions by LNG-IUS outcomes in the women enrolled, and the women who received the LNG-IUS using binomial exact calculations. Chi-square for comparison of proportions, with Fisher's exact test where appropriate, and parametric and non-parametric ttests for comparison of means and medians were used to describe differences between women who did and did not receive the LNG-IUS, and women who did and did not have a spontaneous expulsion of the LNG-IUS.

\section{Results}

Forty women enrolled from July 2009 through February 2010. One woman did not deliver at UNC Women's Hospital. Five women (13\%) were not medically eligible to receive the LNG-IUS due to cesarean delivery $(n=4)$ and preeclampsia $(n=1)$. Three women did not 
receive the LNG-IUS because their births were missed by study staff. One woman changed her mind and decided against receiving the LNG-IUS when undergoing postpartum assessment by study staff and one woman had a failed attempted insertion due to uterine position. Thus, 29 of the original 40 women (73\%) received the LNG-IUS prior to discharge from the hospital.

The baseline characteristics of the women were similar between the women who received the LNG-IUS and those who did not (Table 1). All 29 women who received the LNG-IUS reported they would recommend placement at this time frame again, despite 12 (41\%) women having had a laceration (all second degree) at birth.

Eleven of the 29 women [38\% (95\% CI 21,58)] who received the LNG-IUS had a spontaneous expulsion. The women who had spontaneous LNG-IUS expulsion were younger (21 years compared to 26) than the women who did not have an expulsion $(\mathrm{p}=0.0082)$. All other demographic and physical characteristics at the time of LNG-IUS placement were similar including number of hours between vaginal birth and IUD insertion, and estimated uterine size and cervical dilation (Table 2). Ten of the expulsions were complete. The one incomplete expulsion was identified when the woman came in for an interim study visit due to reported discomfort and the LNG-IUS was visualized protruding from the cervix. Ten expulsions occurred before the six-week follow-up visit, and one occurred one week after the six-week follow-up visit. Six of the expulsions occurred within 14 days of LNG-IUS placement.

Two women were lost to follow-up after insertion despite multiple attempts at contact. After the first follow-up, one woman moved out-of-state and one requested LNG-IUS removal. One woman was lost to follow-up after the second visit.

Five participants contacted study staff prior to the first follow-up visit requesting to be seen for string trimming. One woman removed her LNG-IUS herself in this time period because the strings were painful and irritating.

\section{Discussion}

Placement of the LNG-IUS after birth but before discharge from the hospital, in a treatment room on the postpartum floor, was acceptable to women. The expulsion rate of $38 \%$ had statistical instability due to the small sample size of this feasibility study. However, our report may assist with individual counseling of women interested in postpartum LNG-IUS placement, and informs future study designs.

The published literature on immediate postpartum LNG-IUS placement, especially more than $6 \mathrm{~h}$ after vaginal birth and after women had left the labor and delivery unit, is limited. Dahlke et al. [6] randomized 46 women to LNG-IUS placement within $10 \mathrm{~min}, 10 \mathrm{~min}$ to 48 $\mathrm{h}$ after, or 6 weeks after vaginal birth. However, 14 of the 15 women randomized to receive the LNG-IUS $10 \mathrm{~min}$ to $48 \mathrm{~h}$ later actually received the LNG-IUS within $30 \mathrm{~min}$, and all women received the LNG-IUS on the labor and delivery unit.

Reported spontaneous expulsions in postpartum LNG-IUS studies have ranged from $10 \%$ to $28 \%$. Although our expulsion rate (38\%) is higher than previous reports, the lower limit of the confidence interval, at $21 \%$, falls within previous reports [7,8,11]. The $38 \%$ 6-month expulsion rate we report also falls within the wide range of reported expulsions in previous studies of CuT380A immediate postpartum placement. One prospective study in Turkey reported an expulsion rate of $70 \%$, whereas another multicenter report estimated an expulsion rate of only $5 \%$ when the IUDs were placed by experienced clinicians $[9,10]$. This wide range of reported expulsion rates underscores the need for additional studies to better 
define the rates of expulsion, and which factors may be associated with success or failure. Reasons for our high expulsion rate may be that none of the authors had prior experience with postpartum IUD insertion, however this could be generalized to a clinical setting in which postpartum IUD insertion is being introduced. An additional important limiting factor in much of the published literature on postpartum IUD placement has been the lack of specific information on the time interval between placenta delivery and IUD insertion [3], the inclusion of the time interval in our data report is strength of our study. One relevant clinical aspect of our study is that we did not collect data on analgesia use during labor and delivery or at the time of LNG-IUS insertion. We did not offer analgesia to the women at the time of LNG-IUS insertion.

In conclusion we found that immediate postpartum placement of the LNG-IUS on a postpartum ward was feasible and acceptable for the women in our study. Our reported expulsion rate of $38 \%$ should be interpreted with caution, and fully considered in the larger context of contraceptive continuation and unwanted pregnancy prevention. The focus of this feasibility study was on the women who received an LNG-IUS, and did not have a spontaneous expulsion. Therefore, our study is limited because we did not follow women who had expulsions to measure whether or not they had an IUD reinserted by their primary care provider. Consequently we did not report the proportion of women using the LNG-IUS at 6 months. The role of reinsertion after a postpartum expulsion may be important in overall IUD use. For instance, even when 24-28\% of women have an LNG-IUS expulsion, many will return for a new LNG-IUS placement, such that use of the LNG-IUS at 6 months will be up to 93\% [7]. Finally, our findings can assist clinicians, and investigators, in identifying an expulsion rate for study design purposes, and for counseling women who may be interested in postpartum placement of the LNG-IUS.

\section{Acknowledgments}

The research was supported by grants from the Society of Family Planning (Grant SPF3-14) and NC TraCS (10KR51036). Dr Stuart is supported by a WRHR Faculty Scholar Award (NIH 5K12 HD050113). These data will be presented at the North American Forum on Family Planning in Washington, DC, October 22-24, 2011.

\section{References}

1. Finer LB, Henshaw SK. Disparities in rates of unintended pregnancy in the United States, 1994 and 2001. PerspectSex ReprodHealth. 2006; 38(2):90-6.

2. Gurtcheff SE, Turok DK, Stoddard G, Murphy PA, Gibson M, Jones KP. Lactogenesis after early postpartum use of the contraceptive implant: a randomized controlled trial. Obstet Gynecol. 117(5): 1114-21. [PubMed: 21508750]

3. Kapp N, Curtis KM. Intrauterine device insertion during the postpartum period: a systematic review. Contraception. 2009; 80:327-36. [PubMed: 19751855]

4. Ogburn JA, Espey E, Stonehocker J. Barriers to intrauterine device insertion in postpartum women. Contraception. 2005; 72:426-9. [PubMed: 16307964]

5. World Health Organization. Medical Eligibility Criteria for Contraceptive Use. Fourth Edition. Geneva, Switzerland: 2004. Available at: http://whqlibdoc.who.int/publications/2004/9241562668.pdf.

6. Department of Health and Human Services. Adapted from the World Health Organization Medical Eligibility Criteria for Contraceptive Use. Fourth Edition. Centers for Disease Control and Prevention; Atlanta: May 28. 2010 U.S. Medical Eligibility Criteria for Contraceptive Use, 2010. 2010Available at: http://www.cdc.gov/mmwr/pdf/rr/rr59e0528.pdf.

7. Chen BA, Reeves MF, Hayes JL, Hohmann HL, Perriera LK, Creinin MD. Postplacental or delayed insertion of the levonorgestrel intrauterine device after vaginal delivery: a randomized controlled trial. Obstet Gynecol. 2010; 16:1079-87. [PubMed: 20966692] 
8. Dahlke JD, Terpstra ER, Ramseyer AM, Busch JM, Rieg T, Magann EF. Postpartum insertion of levonorgestrel intrauterine system at three time periods: a prospective randomized pilot study. Contraception. DOI: 10.1016/.2011.

9. Morrison C, Waszak C, Katz K, Diabate F, Mate EM. Clinical outcomes of two early postpartum IUD insertion programs in Africa. Contraception. 1996; 53:17-21. [PubMed: 8631184]

10. Eroglu K, Akkuzu G, Vural G, et al. Comparison of efficacy and complications of IUD insertion in immediate postplacental/early postpartum period with interval period: 1 year follow-up. Contraception. 2006; 74:376-81. [PubMed: 17046378]

11. Hayes JL, Cwiak C, Goedken P, Zieman M. A pilot clinical trial of ultrasound-guided postplacental insertion of a levonorgestrel intrauterine device. Contraception. 2007; 76:292-6. [PubMed: 17900440]

12. O'Hanley K, Huber DH. Postpartum IUDS: keys for success. Contraception. 1992; 45(4)::351-61. [PubMed: 1516367] 
Table 1

Baseline characteristics by whether or not women received the IUD in the hospital

\begin{tabular}{lccc}
\hline Characteristic & \multicolumn{2}{c}{ Received LNG-IUS postpartum in hospital } & p-value \\
\cline { 2 - 3 } & $\underline{\text { Yes }(\mathbf{n}=\mathbf{2 9})}$ & $\underline{\mathbf{N o}}(\mathbf{n}=\mathbf{1 1})$ & \\
\hline Age & $23[21,29]$ & $23[21,25]$ & 0.47 \\
Gravidity & $3[1,4]$ & $2[1,4]$ & 0.64 \\
Parity & $1[0,2]$ & $0[0,1]$ & \\
EGA at study enrollment & $35[32,37]$ & $31[29,36]$ & 0.19 \\
EGA at start of prenatal care & $11[8,21]$ & $10[8,15]$ & 0.78 \\
Race & & & 0.64 \\
White & $17(59)$ & $7(64)$ & \\
Black & $9(31)$ & $2(18)$ & \\
Other & $3(10)$ & $2(18)$ & \\
Hispanic ethnicity & $7(24)$ & $2(18)$ & 0.69 \\
Married or cohabitating & $9(31)$ & $3(27)$ & 0.82 \\
Employed & $2(7)$ & $1(10)$ & 0.02 \\
Prior use of contraceptive & & & \\
Intrauterine contraception & $9(31)$ & $3(27)$ & 0.82 \\
Oral contraception & $21(75)$ & $6(55)$ & 0.36 \\
Transdermal patch & $6(21)$ & $1(10)$ & 0.37 \\
Vaginal ring & $1(4)$ & $0(0)$ & 0.53 \\
Depot medroxyprogesterone acetate & $11(40)$ & $5(45)$ & 0.73 \\
Condom & $23(82)$ & $8(72)$ & 0.51 \\
\hline
\end{tabular}

$\mathrm{N}(\%)$ unless otherwise stated; Median [1 ${ }^{\mathrm{st}}, 3^{\mathrm{rd}}$ quartiles]; EGA: Estimated gestational age 
Table 2

Characteristics of the 29 women who received the LNG-IUS, at insertion, by whether or not the LNG-IUS expelled during the study period

\begin{tabular}{lccc}
\hline Characteristic & Not expelled (N=18) & Expelled (n=11) & p-value \\
\hline Age & $26[23,29]$ & $21[21,24]$ & $<0.01$ \\
Gravidity & $3[2,4]$ & $3[1,4]$ & 0.61 \\
Parity & $2[1,2]$ & $1[0,2]$ & 0.34 \\
Married or cohabitating & $7(39)$ & $2(18)$ & \\
Time interval IUD insertion (h) & $19[11,24]$ & $23[12,27]$ & 0.43 \\
Cervix dilation (cm) & $3[2,4]$ & $2[2,4]$ & 0.18 \\
Uterine size at insertion (wks) & $18[16,18]$ & $19[18,20]$ & 0.10 \\
Received perineal lacerations at delivery & $5(28)$ & $7(64)$ & 0.06 \\
Reported discomfort, scale 1-10 & $1[1,3]$ & $3[1,6]$ & 0.21 \\
Would recommend to a friend at insertion & $18(100)$ & $11(100)$ & \\
Method of insertion & & & 0.59 \\
Hand & $2(11)$ & $2(20)$ & \\
Forceps & $17(89)$ & $8(80)$ & \\
Inserter & 0 & 0 & \\
IUD in place at study exit & & & \\
Yes & 17 & 0 & \\
No & 1 & 11 & \\
\hline
\end{tabular}

Data represented as $\mathrm{n}(\%)$ with $95 \%$ confidence interval unless otherwise stated, or median [1 ${ }^{\mathrm{st}}, 3^{\mathrm{rd}}$ quartiles]. 